\title{
Random flows and diagnostics of turbulence in the high latitude cirrus ${ }^{\star}$
}

\author{
S. N. Shore ${ }^{1}$, T. N. LaRosa ${ }^{2}$, R. J. Chastain ${ }^{3}$, and L. Magnani ${ }^{3}$ \\ 1 Dipartmento di Fisica "Enrico Fermi”, Università di Pisa, via Buonarroti 2, Pisa 56127 and INFN - Sezione di Pisa, Italy \\ e-mail: shore@df.unipi.it \\ 2 Department of Biological \& Physical Sciences, Kennesaw State University, 1000 Chastain Road, Kennesaw, GA 30144, USA \\ e-mail: ted@avatar.kennesaw.edu \\ 3 Department of Physics and Astronomy, University of Georgia, Athens GA 30602, USA \\ e-mail: loris@physast.uga.edu
}

Received 1 August 2005 / Accepted 7 July 2006

\section{ABSTRACT}

\begin{abstract}
Aims. We present an analysis of the exceptionally turbulent velocity field in the high Galactic latitude cirrus cloud MBM 3. As in the other translucent clouds in our study (MBM 16 and MBM 40), there is no evidence for internal star formation. However, the large scale velocity variation in this cloud is more pronounced.

Methods. We have mapped the cloud in ${ }^{12} \mathrm{CO}$ and ${ }^{13} \mathrm{CO}(1-0)$ at high spatial $(0.03 \mathrm{pc})$ and velocity $\left(0.06 \mathrm{~km} \mathrm{~s}^{-1}\right)$ resolution. We constructed several velocity probability density functions (PDFs), estimated the turbulent transfer rate, and analyzed the linewidths as a function of the size of randomly chosen regions within the cloud.

Results. We find strong shear flows throughout the cloud that can easily power the turbulent motions. The wings of the PDFs are well approximated by a lorentzian distribution. Such distributions, related to Lev̀y processes that are well known to be produced by correlated processes, are an unambiguous diagnostic of the turbulent intermittency.

Conclusions. We find that the linewidth-size relation frequently used to indicate the role of turbulence in molecular clouds is not an unambiguous signature of its presence.
\end{abstract}

Key words. turbulence - ISM: kinematics and dynamics - ISM: clouds - ISM: structure

\section{Introduction}

During the past decade, millimeter Doppler imaging studies, infrared observations, and innovative statistical analyses have been complemented by an impressive body of numerical simulations to build a compelling case for the fundamental importance of turbulence as the principal agent producing complex density and velocity structures in atomic and molecular clouds in the interstellar medium (ISM) (see e.g. Larson 2003; Elmegreen \& Scalo 2004; Scalo \& Elmegreen 2004; Mac Low \& Klessen 2004, for recent comprehensive reviews). Its presumed role in regulating the star formation process has also been the subject of intense study (for recent conferences see Corbelli et al. 2005; Gomez de Castro et al. 2004; Falgarone \& Passot 2003). Yet the signature(s) of the specific process(es) by which turbulence is driven and maintained in the highly dissipative ISM remain poorly understood.

The conceptual foundation of turbulence is a cascade of energy from large injection scales to small scales where it is dissipated (e.g., Tennekes \& Lumley 1972; McComb 1992). However, an immediate astrophysical complication is that in the ISM, energy can be injected on many scales ranging from galactic scale dynamics (e.g. rotation and spiral density waves) to individual stellar processes such as explosions, jets, H II regions and winds. In an attempt to avoid these effects, we continue to study simpler regions, the cirrus clouds that have never

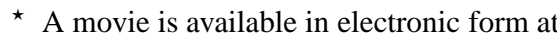
http://www.edpsciences.org undergone star formation. Most of these clouds exhibit the full range of turbulent signatures but are not self-gravitating and lack internal sources for the observed superthermal motions. The infrared cirrus (Low et al. 1984) traces both atomic and molecular gas. It shows a very tangled filamentary structure. Quantitatively, its emissivity has been fit with a power spectrum of index of 2.8 to 3.2 (Gautier et al. 1992) and its filling actor has been modeled as a a fractal with dimension of 1.8 (Bazell \& Dessert 1990). Such measurements usually imply structuring by turbulence. Translucent molecular clouds have been found to be associated with a portion of the infrared cirrus and usually correspond to regions of higher emissivity, indicating higher dust column density but not higher temperatures (Weiland et al. 1986; Schlegel et al. 1999). These clouds tend to be nearby ( $100 \mathrm{pc})$, low mass ( $\sim 10$ to $50 M_{\odot}$ ), unbound, transient objects (e.g., Magnani et al. 1993). However, multi-transition ${ }^{12} \mathrm{CO}$ studies indicate that the bulk of the mass in these clouds is in cold $(<10 \mathrm{~K})$ high density $\left(10^{4}\right.$ to $\left.10^{5} \mathrm{~cm}^{-3}\right)$ small $(\sim 1000 \mathrm{AU})$ clumps and filaments (Falgarone et al. 1998; Ingalls et al. 2000).

In a previous study (Shore et al. 2003, hereafter SMLM03) we presented IRAS, $\mathrm{H} \mathrm{I},{ }^{12} \mathrm{CO}$ and ${ }^{13} \mathrm{CO}$ maps of the high latitude translucent cloud MBM40. We found that this cirrus cloud appears to be condensing out of the atomic medium due to a thermal instability, evidence for which is that the $\mathrm{CO}$ peak is surrounded by a hole in the atomic emission and the velocity field of the molecular gas followed the same shear flow as the atomic gas. No evidence for shocks was found and, since the observed velocity shears can easily supply the energy to drive turbulent 
motions, we suggested that a shear flow instability is driving the turbulence in this cloud. In this paper we investigate a similar translucent cloud MBM3 (see Magnani et al. 1985). It was selected for detailed study based on the survey by Magnani et al. (2000) who found exceptionally broad CO $\left(\sim 5 \mathrm{~km} \mathrm{~s}^{-1}\right)$ lines along several lines of sight in the cloud, making it an intriguing target for a dynamical analysis. To that end we have mapped MBM3 in the ${ }^{12} \mathrm{CO}(1-0)$ and ${ }^{13} \mathrm{CO}(1-0)$ transitions with high spatial and velocity resolution.

Our analysis concentrates on using velocity fluctuations to obtain a variety of probability distribution functions (PDFs) for the dynamics and to obtain estimates of the turbulent dissipation rate. These methods have long been used in engineering studies and have only relatively recently become a standard tool in studies of stellar convection and interstellar dynamics (see Elmegreen \& Scalo 2004). This is principally because cosmic objects are more complicated and the observations far less comprehensive. In the laboratory, PDFs are used to separate random (Gaussian) motions from truly turbulent flows (Sreevivasan \& Antonia 1997; Minier \& Peiran 2001). When applied to molecular clouds, for instance, only the radial velocity is available and some care must be exercised in forming an analogy between the laboratory and extraterrestrial measurements. In a controlled flow, a PDF formed from velocity differences taken at two points along the direction of the flow (which we will refer to as the shift $P D F$ ) removes the mean (or systematic) motion and recovers a Lagrangian description of the fluid but this is taken in time at the two locations around the zeroed centroid velocity. This is harder to do when only one component of velocity and two of displacement (both orthogonal to the line of sight) are measured and with only a snapshot. Notwithstanding these limitations, non-Gaussian velocity wings are found usually on short lags, implying the existence of small scale regions of correlated motions.

This behavior was found in simulations of decaying turbulence for astrophysically relevant conditions by Lis et al. (1996). They associated the broad wings with very compact regions of high vorticity, as often detected in laboratory-scale flows. In one of the most extensive mapping studies, Miesch et al. (1998, hereafter MSB99) did indeed find non-Gaussian PDFs at small lags. They also found (as we will later discuss) small scale structures that mimic some properties of turbulent media but they did not associate these fluctuations with any particular small scale dynamics. Their velocity difference maps actually show correlated filamentary structures but not with the degree of organization expected from the simulations. This is not too surprising since the simulations modeled decaying turbulence while the observations probed active, driven flows. Ossenkopf \& Mac Low (2002) also studied continuously driven turbulence but without shear flows. MSB99 argued that the environment of a star forming cloud is stirred continuously and may be dominated by shocks. In contrast, Pety \& Falgarone (2003) studied two starless cores; they also found non-Gaussian wings of their PDFs that they interpreted as tracing regions of high vorticity.

It is important to reconcile these differences and determine if different signatures emerge from star-forming and non-starforming clouds and what forms the dissipation may take.

\section{Observations}

The ${ }^{12} \mathrm{CO}$ and ${ }^{13} \mathrm{CO}$ data were obtained simultaneously using the Five Colleges Radio Observatory (FCRAO) 14-m radio telescope ${ }^{1}$ in April and May of 2003. The cloud was mapped using the on-the-fly (OTF) technique in combination with the SEQUOIA focal plane array receiver. With OTF mapping, the antenna is scanned continuously across the source and data are dumped at a fast enough rate to prevent significant beam smearing. By continuously taking data and allowing the spatial filter and processing software to appropriately place each dumped scan within the map, the overhead time to move and point the antenna is greatly reduced. For the MBM03 observations, the SEQUOIA focal plane array consisted of 32 single sideband elements arranged in dual-polarized $4 \times 4$ pattern that extends over a $5.9^{\prime} \times 5.9^{\prime}$ region referred to as a "footprint". The CO map is sampled at better than the Nyquist rate (20" sampling with a resolution of $\left.47^{\prime \prime}\right)$. Both polarizations are observed simultaneously. The final map was made in thirty $12 \times 12$ sections for a total spatial coverage of $1.2^{\circ} \times 1.0^{\circ}$ in $(l, b)$. For the OTF map, the data were taken in position-switched mode with the reference position located at $l=131^{\circ}, b=-45^{\circ}$.

Because the FCRAO spectrometer allows for simultaneous observations of both the ${ }^{12} \mathrm{CO}(1-0)$ and ${ }^{13} \mathrm{CO}(1-0)$ transitions, the dual 1024-channel autocorrelator was set up to observe a $25 \mathrm{MHz}$ bandwidth centered on the rest frequencies of the ${ }^{12} \mathrm{CO}$ and ${ }^{13} \mathrm{CO}$ lines (115.271 and $110.201 \mathrm{GHz}$, respectively). At these frequencies, the bandwidth corresponds to a velocity coverage of $65 \mathrm{~km} \mathrm{~s}^{-1}$ for ${ }^{12} \mathrm{CO}$ and $68 \mathrm{~km} \mathrm{~s}^{-1}$ for ${ }^{13} \mathrm{CO}$, and velocity resolutions per channel of 0.063 and $0.066 \mathrm{~km} \mathrm{~s}^{-1}$, respectively. The angular resolution of the 14-m antenna is $47^{\prime \prime}$ at $115 \mathrm{GHz}$ (corresponding to a spatial resolution of $0.03 \mathrm{pc}$ at the estimated distance of $130 \mathrm{pc}$ (see Sect. 3.2) and 49 arcsec at $110 \mathrm{GHz}$.

At a dumping rate of once per second and typical $T_{\text {sys }}$ values of $\sim 800 \mathrm{~K}$, adding both polarizations together resulted in typical rms noise values of $\leq 0.2 \mathrm{~K}$ in units of antenna temperature, $T_{\mathrm{A}}$. To convert $T_{\mathrm{A}}$ to the radiation temperature, $T_{\mathrm{R}}$, appropriate values for the forward-scattering add spillover efficiency, $\eta_{\text {fss }}$, and source filling factor, $\eta_{\mathrm{c}}$, must be used. For the 14-m antenna at 110 and $115 \mathrm{GHz}, \eta_{\mathrm{fss}}=0.45$ (Mark Heyer, private communication). The factor $\eta_{\mathrm{c}}$ is taken to be unity because the angular extent of MBM03 is much larger than the angular resolution of the telescope.

The line profiles in MBM 3 show that there are two clouds along the line of sight. One component, centered on an LSR radial velocity of $-5.6 \mathrm{~km} \mathrm{~s}^{-1}$, has a velocity width of about $4 \mathrm{~km} \mathrm{~s}^{-1}$ (FWHM); the other, centered on $\approx-1.5 \mathrm{~km} \mathrm{~s}^{-1}$, is narrower, $2.7 \mathrm{~km} \mathrm{~s}^{-1}$ (FWHM), and substantially weaker. The broader component is detected from the entire structure while the narrower component comes from only the southern part of the cloud and is likely a separate, but associated, structure superimposed on the broader compent. We will here concentrate in the remainder of the paper only on the more widely distributed component which we will refer to as the "northern" component. We computed the intensity weighted velocity centroid at each pixel. This weighting avoids any bias that can be introduced by parametric fitting. We also kept only those pixels with a signal to noise ratio of at least 10 . In all this gave us approximately 23500 points.

The individual spectra all contain a small radio interference (RFI) spike at $v_{\mathrm{LSR}}=0 \mathrm{~km} \mathrm{~s}^{-1}$ produced by the autocorrelator. The RFI occurs at a fixed sky frequency and moves in $v_{\text {LSR }}$ according to the Doppler tracking velocity of the telescope

1 FCRAO is supported in part by National Science Foundation under grant AST94-20159 and is operated with permission of the Metropolitan District Commission, Commonwealth of Massachusetts. 


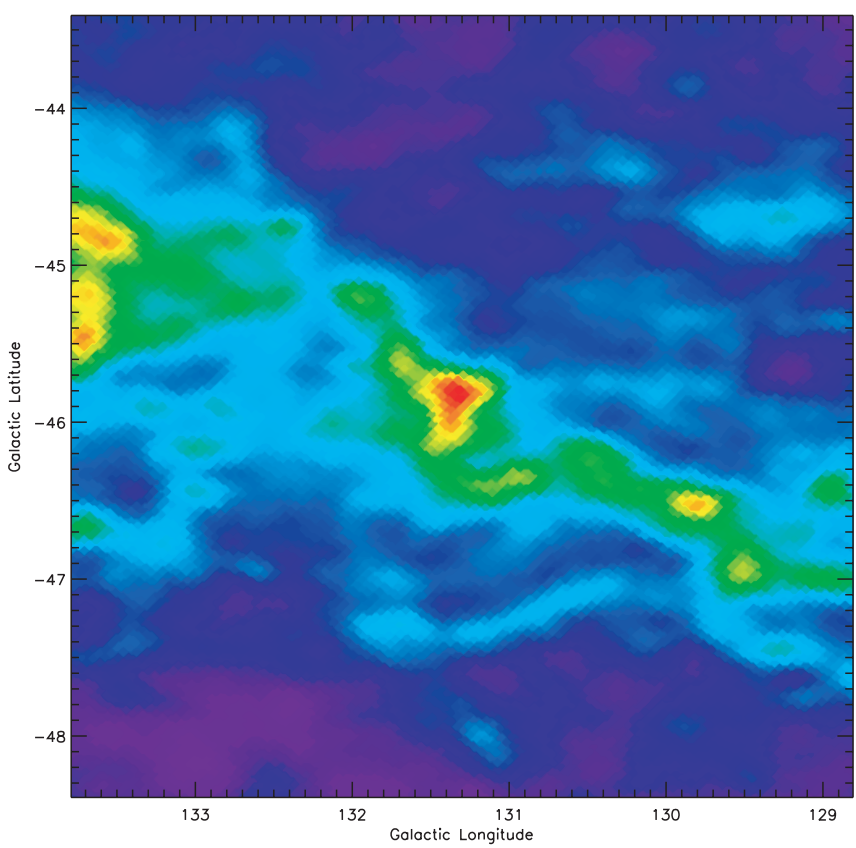

Fig. 1. Large-scale IRAS 100 micron map from the IRAS Sky Survey Atlas (Wheelock et al. 1994) centered on MBM 3 (the triangularly shaped feature in the center of the map). The filamentary cirrus structure containing MBM 3 extends eastward into MBM 4 at the map edge. The 100 micron surface brightness increases linearly from 2.52 to $12.28 \mathrm{MJy} / \mathrm{ster}$, and the resolution is $\sim 4^{\prime}$.

(Mark Heyer, private communication). For the observations described in this paper, the RFI spike was away from the bulk of the cloud emission which extends from -11 to $-1.8 \mathrm{~km} \mathrm{~s}^{-1}$. However, a smaller component was present in some of the spectra at $v_{\mathrm{LSR}} \sim+1 \mathrm{~km} \mathrm{~s}^{-1}$. Since we have not included this cloud component in our analysis no attempt was made to reduce the interference.

\section{Analysis}

\subsection{Infrared and molecular gas morphology}

Schlegel et al. (1998, hereafter SFD) produced a series of dust maps based on the IRAS and COBE databases. Their $E(B-$ $V)$ maps are good tracers of translucent molecular gas (e.g., Magnani et al. 2003) and provide more reliable gas column density estimates than maps of the IRAS flux. In Fig. 1 we show the IRAS $100 \mu$ image and in Fig. 2 we show the SFD dust maps centered on MBM 3 over $5^{\circ} \times 5^{\circ}$ and $1.2^{\circ} \times 1.2^{\circ}$ regions, respectively.

It is clear from the larger map that the translucent cloud (the triangularly shaped structure in the center of the map) is part of a large scale filamentary structure. Even larger images than that shown in Fig. 1 indicate that the filament containing MBM 3 extends over about $20 \mathrm{pc}$ (more than $10 \mathrm{deg}$ ) and includes MBM 4 (the edge of which is visible in the eastern portion of the map in Fig. 1). The velocity-integrated ${ }^{12} \mathrm{CO}(1-0)$ antenna temperature [defined to be $W\left({ }^{12} \mathrm{CO}\right)$ for the remainder of the paper] is shown in Fig. 3. The composite spectrum for the cloud (see Fig. 4) indicates that it is made up of two separate, principal, velocity components; one extending from -10 to $-1.8 \mathrm{~km} \mathrm{~s}^{-1}$ and the other from -1.8 to $+3 \mathrm{~km} \mathrm{~s}^{-1}$. The spectral profiles are even more complicated for individual lines of sight as can be seen in Fig. 5. Spatially, the more negative velocity component is primarily in the northern portion of the map and is depicted by

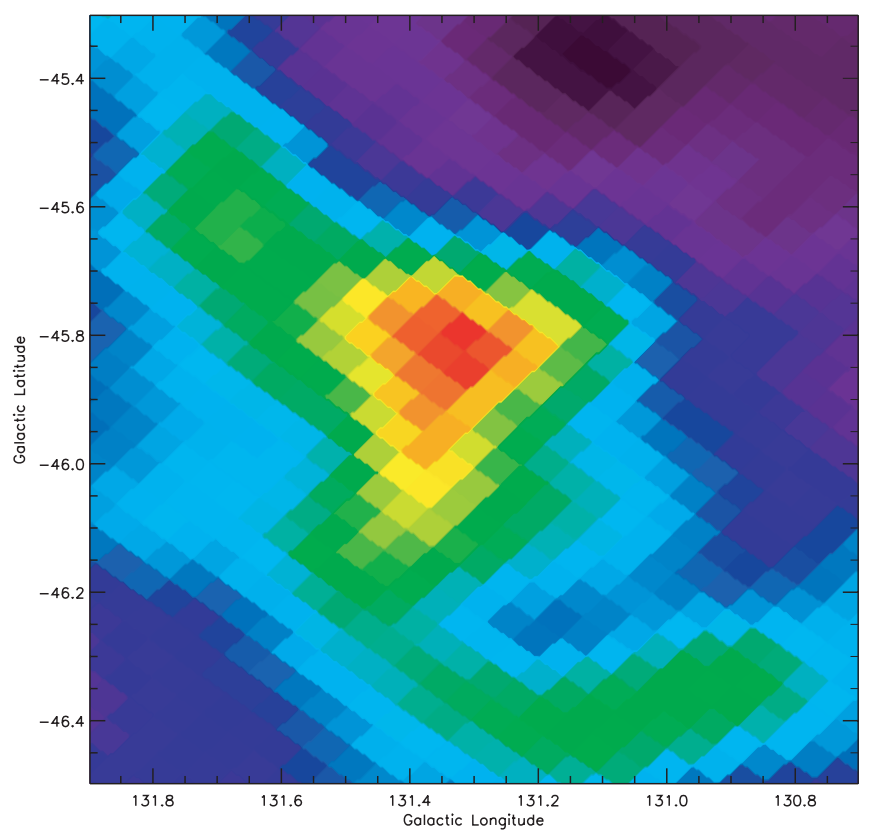

Fig. 2. $E(B-V)$ map from Schlegel et al. (1998) centered on MBM 3 (compare to Fig. 3). The $E(B-V)$ range is linear from 0.06 to $0.23 \mathrm{mag}$ and the resolution is $\sim 6^{\prime}$.

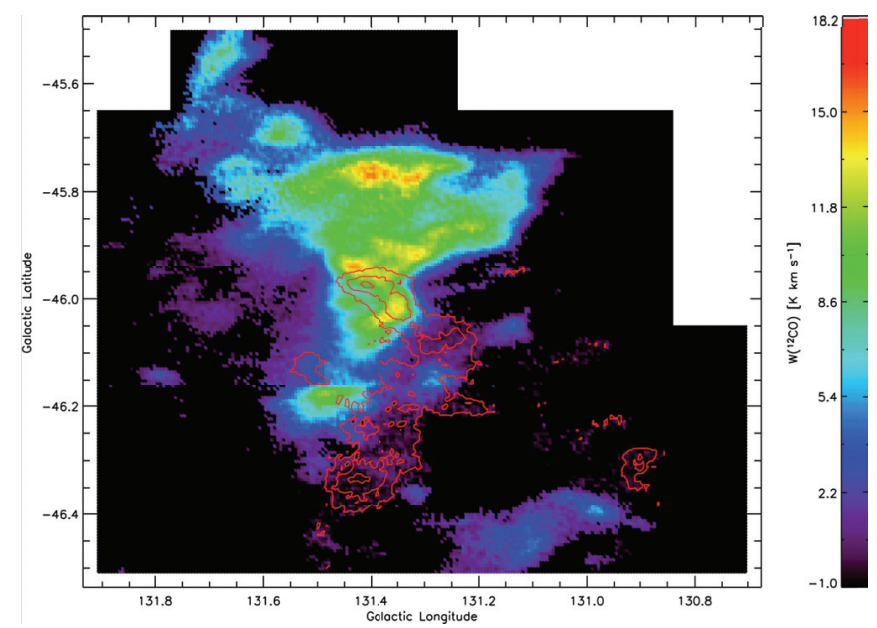

Fig. 3. Color image of the velocity-integrated ${ }^{12} \mathrm{CO}(1-0)$ emission $W\left({ }^{12} \mathrm{CO}\right)$ for MBM 3 over the range -10 to $-1.8 \mathrm{~km} \mathrm{~s}^{-1}$. The color bar shows the value of $W\left({ }^{12} \mathrm{CO}\right)$ in units of $\left[\mathrm{K} \mathrm{km} \mathrm{s}^{-1}\right]$. A separate velocity component $\left(-1.8\right.$ to $\left.3 \mathrm{~km} \mathrm{~s}^{-1}\right)$ is shown by the red line contours. These levels are at $2.5,4$, and $5.5 \mathrm{~K} \mathrm{~km} \mathrm{~s}^{-1}$. That MBM 3 is made up of at least two distinct velocity components is clear from the spectra (see Fig. 4). The CO map is sampled at better than the Nyquist rate $\left(20^{\prime \prime}\right.$ sampling with a resolution of $47^{\prime \prime}$ ). The "northern" portion of the cloud referred to in the text is the color map.

the color portion of Fig. 3, while the other velocity component is in the south and is denoted by red line contours. A comparison between Figs. 2 and 3 shows that the close morphological relationship between the SFD dust data and $W\left({ }^{12} \mathrm{CO}\right)$.

The bulk of CO emission in MBM 3 comes from a region about 1 to $2 \mathrm{pc}$ in size. In the plane of the sky the integrated ${ }^{12} \mathrm{CO}$ appearance of the cloud is vertically extended with an anvil-like morphology. However, the appearance of the cloud changes considerably with velocity. A striking velocity feature is the banding of the image on a scale of about $0.6 \mathrm{pc}\left(16^{\prime}\right)$, a point to which we will return below. 


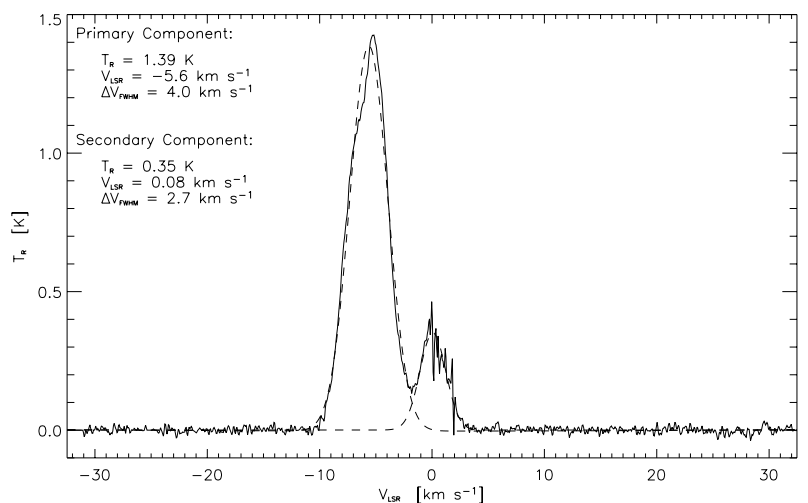

Fig. 4. Summed ${ }^{12} \mathrm{CO}$ spectrum from the mapped region. Two principal components are fit by Gaussians with the parameters shown directly in the figure.
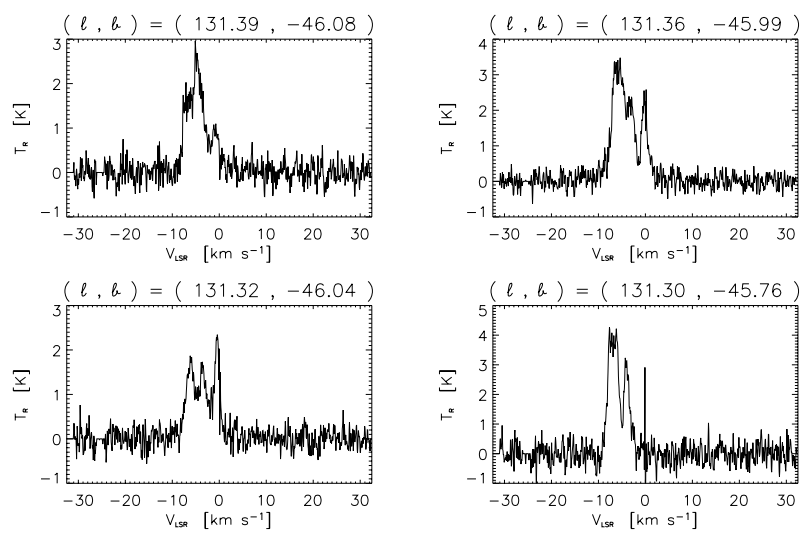

Fig. 5. Examples of individual ${ }^{12} \mathrm{CO}(1-0)$ line profiles for four random lines of sight in MBM 3.

\subsection{The mass of the cloud}

The mass of the cloud can be expressed in terms of the distance, cloud area, and average $N\left(\mathrm{H}_{2}\right)$ as

$M=5.8 \times 10^{-3} A d^{2} N_{21} M_{\odot}$,

where $A$ is the area of the cloud in square degrees, $d$ is the distance in pc, and $N_{21}$ is the the average $\mathrm{H}_{2}$ column density in units of $10^{21} \mathrm{~cm}^{-2}$. If we assume a distance to the cloud of $130 \mathrm{pc}(\mathrm{a}$ range of $90-170 \mathrm{pc}$ is proposed by Lallement et al. 2003) and a CO-emitting area of 0.29 square degrees (Chastain 2005), equation 1 becomes $M=28 N_{21} M_{\odot}$. The value of $N\left(\mathrm{H}_{2}\right)$ can be obtained directly from ${ }^{12} \mathrm{~W}(\mathrm{CO})$ if the $\mathrm{CO}-\mathrm{H}_{2}$ conversion factor is known, from ${ }^{13} \mathrm{CO}(1-0)$ data if the ${ }^{13} \mathrm{CO}-\mathrm{H}_{2}$ conversion factor is known, and it can also be independently derived from the SFD dust data assuming that the standard gas-to-dust relation of Bohlin et al. (1978) holds. Chastain (2005) derives the mass of MBM 3 by using the ${ }^{13} \mathrm{CO}$ data and LTE assumptions to obtain $N\left({ }^{13} \mathrm{CO}\right)$, and then using the empirically derived relationship between $N\left({ }^{13} \mathrm{CO}\right)$ and $N\left(\mathrm{H}_{2}\right)$ to obtain the latter quantity (Dickman 1978). The average value of $N\left(\mathrm{H}_{2}\right)$ over the cloud derived from this technique is $7.3 \pm 3.2 \times 10^{20} \mathrm{~cm}^{-2}$, which leads to a molecular cloud mass of $20 \pm 9 M_{\odot}$. Like virtually all high-latitude molecular clouds, MBM 3 is contained within a filament or extended structure of atomic hydrogen. The LeidenDwingeloo survey (Hartmann \& Burton 1997) can be used to determine the average value of $N(\mathrm{HI})$ in the direction of the cloud, $4.3 \times 10^{20} \mathrm{~cm}^{-2}$. This contributes another 12 solar masses to the overall cloud mass.

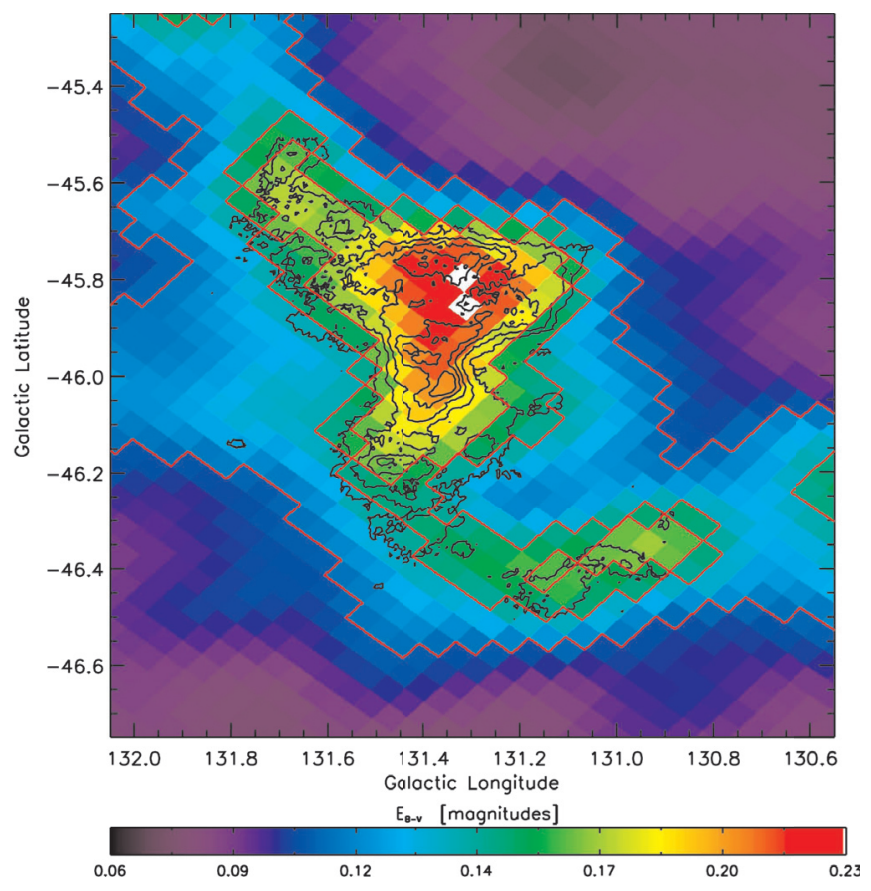

Fig. 6. Color image of the SFD $E(B-V)$ map with color bar of the scale at the bottom. The $\mathrm{W}^{12} \mathrm{CO}$ contours (i.e., the data shown in Fig. 3 ) are plotted in black and the contour lines for $E(B-V)=0.10,014$, and 0.16 mag are shown in red.

An independent estimate of the cloud mass can be obtained from the SFD $E(B-V)$ maps and the empirical relation between this quantity and $N\left(\mathrm{H}_{\text {total }}\right)$ established by Bohlin et al. (1978) for diffuse and translucent lines of sight towards early type stars. Figure 6 shows the $W\left({ }^{12} \mathrm{CO}\right)$ contours above the $2.1 \mathrm{~K} \mathrm{~km} \mathrm{~s}^{-1}$ level plotted in black over the SFD $E(B-V)$ image of the MBM 3 region. Color excess contours of $0.10,0.14$, and 0.16 mag are plotted in red. As can be seen from the figure, $W\left({ }^{12} \mathrm{CO}\right)$ is well correlated spatially with $E(B-V)>0.14$ mag. By including the contribution from all regions with $E(B-V)>0.14$ mag, the mass estimate including both atomic and molecular gas is $54 M_{\odot}$ (see Chastain 2005 for details), in good agreement with the estimate based on the $\mathrm{CO}$ lines.

In summary, the molecular mass of MBM 3 likely lies in the 20-40 $M_{\odot}$ range, with an additional $10-15 M_{\odot}$ in the form of associated atomic hydrogen.

\subsection{Integrated line profiles and optical depths}

In Fig. 7 we show the ${ }^{12} \mathrm{CO}$ (dashed line) and ${ }^{13} \mathrm{CO}$ (solid line) spectral line profiles averaged over the entire cloud. Under the LTE approximation, we can determine the average optical depth of the ${ }^{13} \mathrm{CO}$ line center. For the primary velocity component (see Fig. 4), the ${ }^{13} \mathrm{CO}$ peak line temperature is $0.52 \mathrm{~K}$, producing a value for $\tau\left({ }^{13} \mathrm{CO}\right)$ of 0.37 . Assuming a local $\left({ }^{12} \mathrm{CO} /{ }^{13} \mathrm{CO}\right)$ ratio of 62 (Langer \& Penzias 1990), we can see that, at line center, the ${ }^{12} \mathrm{CO}(1-0)$ transition is optically thick. However, as one moves away from the line center, the value of $T_{\mathrm{R}}\left({ }^{12} \mathrm{CO}\right) / T_{\mathrm{R}}\left({ }^{13} \mathrm{CO}\right)$, increases from 2.7 to values greater than 20 . In these regimes, the ${ }^{13} \mathrm{CO}$ optical depth drops to $\sim 0.01$ and the ${ }^{12} \mathrm{CO}$ emission becomes optically thin. Figure 8 shows the ratio $W\left({ }^{12} \mathrm{CO}\right) / W\left({ }^{13} \mathrm{CO}\right)$ for the 3588 lines of sight with a detected ${ }^{13} \mathrm{CO}$ line. As is clear from the figure, the southern portion of the cloud is more optically thin as a whole than the northern portion. We note that the optical depth plotted in Fig. 8 


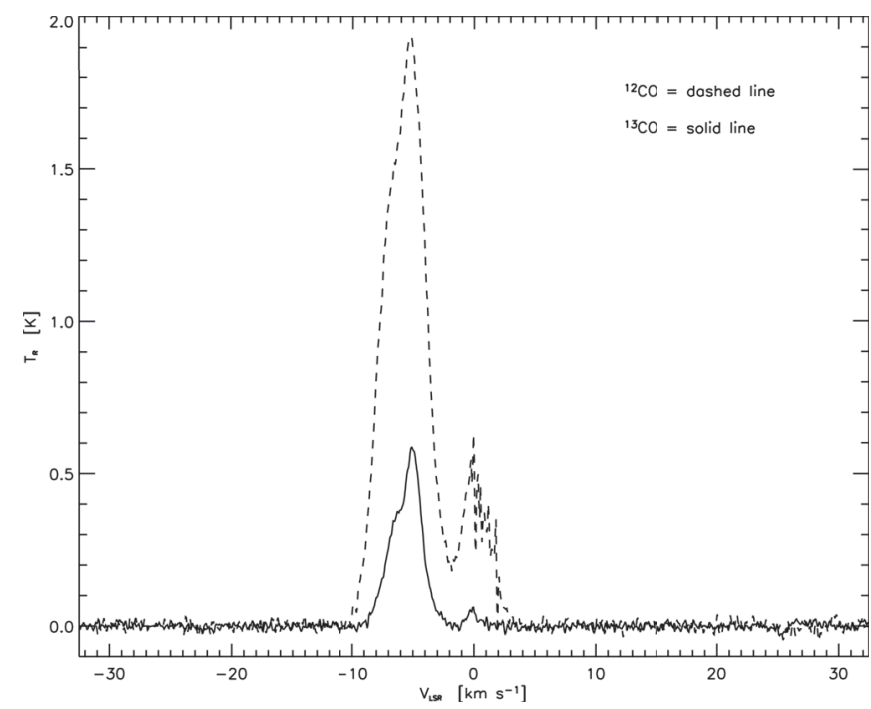

Fig. 7. The average line profile for the ${ }^{13} \mathrm{CO}(1-0)$ transition (solid line) plotted over the stronger ${ }^{12} \mathrm{CO}(1-0)$ average spectrum. The ${ }^{13} \mathrm{CO}$ profile is averaged over 3588 lines of sight with evident signal while the ${ }^{12} \mathrm{CO}$ spectrum is averaged over 13065 lines of sight.

is for the line center. As Fig. 7 makes clear, away from the line center, the ${ }^{12} \mathrm{CO}$ is thin. Thus, at all positions in the cloud, the optical depth varies with velocity and the line wings are optically thin.

\subsection{Doppler imaging}

In Fig. 9 we show velocity channel maps of the cloud. The panels run from upper left to lower right, beginning at $-10.1 \mathrm{~km} \mathrm{~s}^{-1}$ in steps of $0.26 \mathrm{~km} \mathrm{~s}^{-1}$; Galactic north is at the top and west is to the right. The northern and southern extrema of the cloud appear at first almost simultaneously but the northern part persists over a wider range of velocities. As the velocity increases narrow bands of emission on an angular scale of $0.2 \mathrm{pc}\left(6^{\prime}\right)$ develop between the extrema. These are not artifacts and persist over about half of the displayed panels, a range of $5 \mathrm{~km} \mathrm{~s}^{-1}$. Beginning at around $-8 \mathrm{~km} \mathrm{~s}^{-1}$, a filamentary feature appears near the center of the cloud that systematically extends and shifts its centroid toward the west and eventually to the north with increasing radial velocity, while the northern part of the cloud expands across the upper part of the map. Also visible in the channel maps, and more obvious in the accompanying movie (see online material), notice that the systematic flows also occur in the northeastern part of the cloud extending toward the east. The symmetry axis of the image also systematically rotates, beginning at the most negative radial velocities nearly vertically in the at the most negative radial velocities and ending tilted toward the northwest.

In Fig. 10 we show the velocity centroid map of the cloud in the velocity range of the principal componment shown in Fig. 4. The spatial distribution of radial velocities is complex and shows several regions of organized, shearing motion. On sizes of about 0.2 to $0.3 \mathrm{pc}$ there are several large scale flows discernible over the entire velocity range of ${ }^{12} \mathrm{CO}$ emission. The ${ }^{13} \mathrm{CO}$ is generally too weak to show such details. The velocity gradients are of order $3.4 \mathrm{~km} \mathrm{~s}^{-1}$ on a scale of about $0.8 \mathrm{pc}\left(22^{\prime}\right)$. The column density and velocity structures are very ordered and anisotropic in MBM 3. It is important to note that the velocity map doesn't record intensity information, which is only discernible by comparing the intensity and velocity maps. Thus regions with large shear are actually regions of relatively low

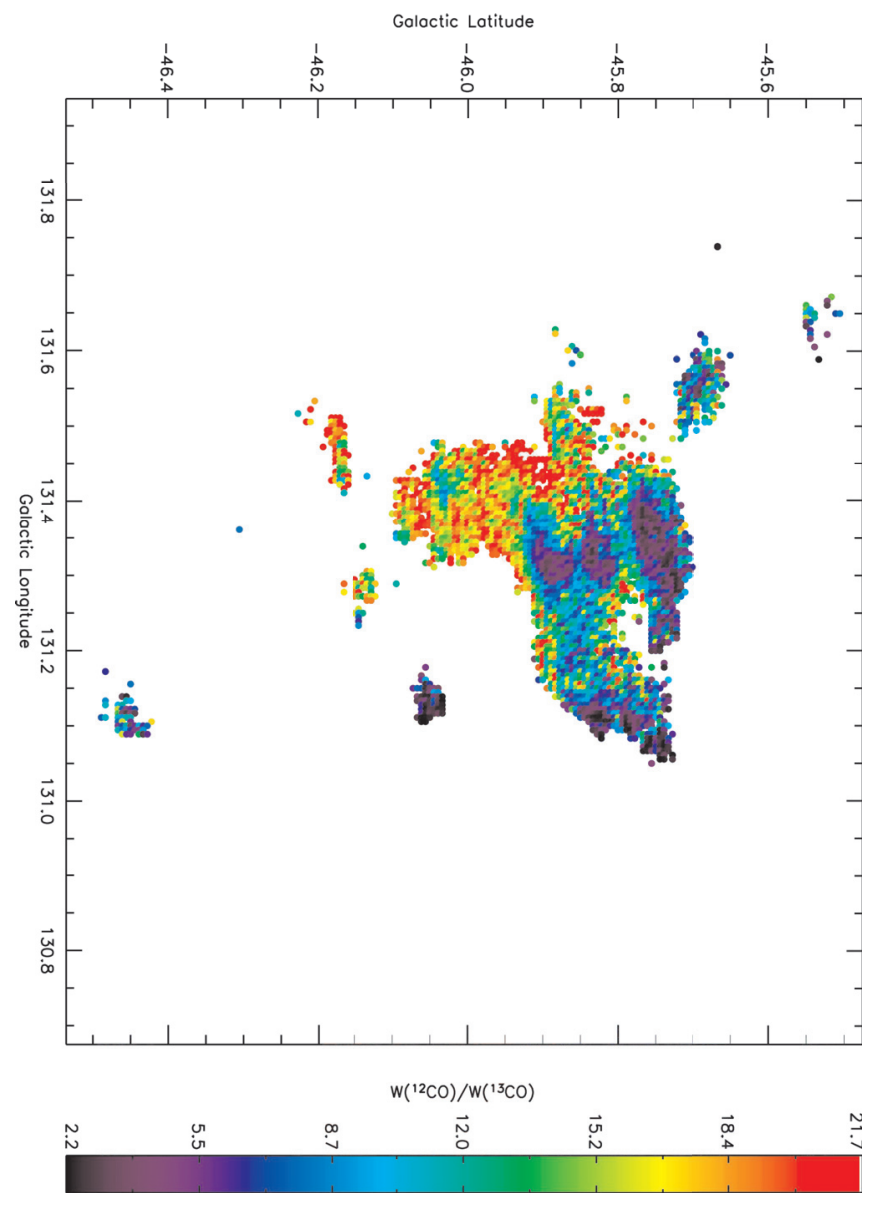

Fig. 8. Color plot of the $W\left({ }^{12} \mathrm{CO}\right) / W\left({ }^{13} \mathrm{CO}\right)$ ratio at each of the 3588 positions within the cloud with a detected ${ }^{13} \mathrm{CO}$ line. The colorbar shows the value of the ratio for a given color.

column density (see again the movie for a clearer view of this phenomenon). In general, this is consistent with the stability of the line profile, the skew is dominated by a comparatively small fraction of the mass of the cloud.

\subsection{Velocity probability distribution functions (PDF)}

The velocity probability distribution function (PDF) is a measure of the frequency distribution of velocities, in effect a histogram. However, in laboratory flows the velocity difference PDFs systematically show non-Gaussian behavior and are a more robust measure of the turbulence (see Miesch et al. 1998, hereafter MSB99; Falgarone \& Phillips 1990). Regardless of the parent distribution for the velocity fluctuations, their PDFs should approach a Gaussian at sufficiently large lag, when the random motions are completely uncorrelated. In astrophysical studies to date several different schemes have been used to compute this diagnostic, correcting for systematic flows by applying different detrending algorithms to the data. For example, Kitamura et al. (1993), Miesch \& Bally (1994), MSB99, LaRosa et al. (1999), and Shore et al. (2003) removed any large scale trends in their maps by computing a mean fitted, or smoothed map, subtracting this from the original data and analyzing the residual fluctuations. In laboratory turbulence studies this corresponds to the usual Reynolds decomposition that focuses on the fluctuations about the mean flow. Detrending is especially important for correlation analyses since large scale gradients will dominate any correlation and mask correlations generated by turbulence. 


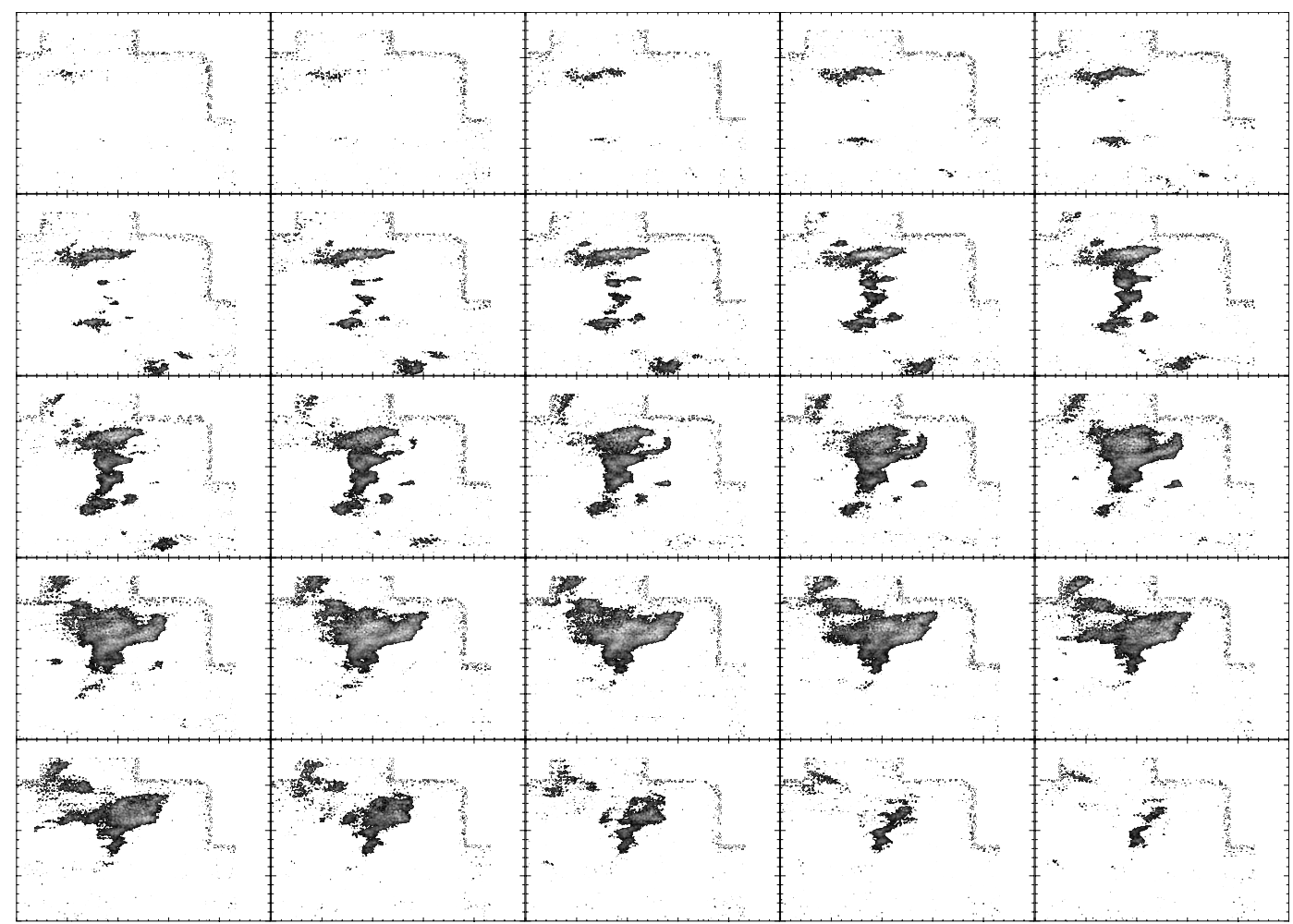

Fig. 9. Channel maps for ${ }^{12} \mathrm{CO}(1-0)$ of MBM 3 in the velocity range $-10.1 \leq v_{\text {rad,LSR }} \leq-3.8 \mathrm{~km} \mathrm{~s}^{-1}$ in steps of $0.26 \mathrm{~km} \mathrm{~s}^{-1}$ (every 4 channels). The maps are $1.2 \mathrm{deg} \times 1 \mathrm{deg}$ in Galactic coordinates, each pixel is 20.1 arcsec (see Fig. 2 for the integrated map). The background level is $T_{\mathrm{A}}=0.2 \mathrm{~K}$ per channel, the peak is $2.7 \mathrm{~K}$. The coordinates are labeled by lags, see text for discussion. The movie (online version) produced from this datacube shows the channel maps in sequence.

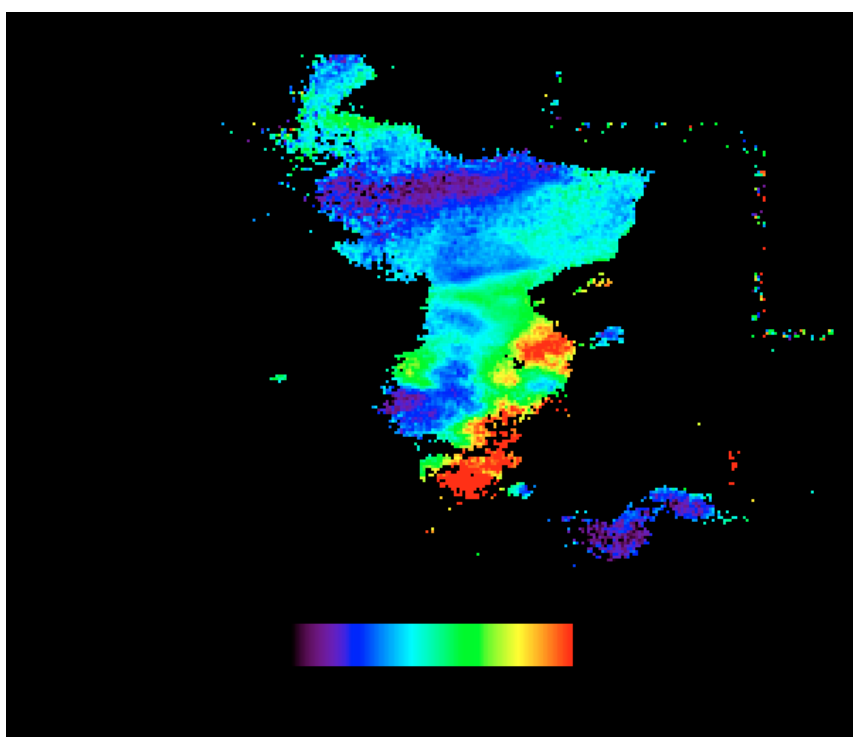

Fig. 10. Velocity centroid map of ${ }^{12} \mathrm{CO}$ in the range -10 to $0 \mathrm{~km} \mathrm{~s}^{-1}$. The color scale corresponds to the range of LSR velocities with red indicating greater (more positive) radial velocity.

Alternatively, Ossenkopf \& Mac Low (2002) and Pety \& Falgarone (2003) did not explicitly remove the large scale trends, arguing that on the scale of their maps such flows are themselves part of the overall cascade and should not be separated. In this study we use both approaches and find they give consistent results.

We used the northern portion of MBM 3, those positions indicated in the channel maps which, in beams, extend from the

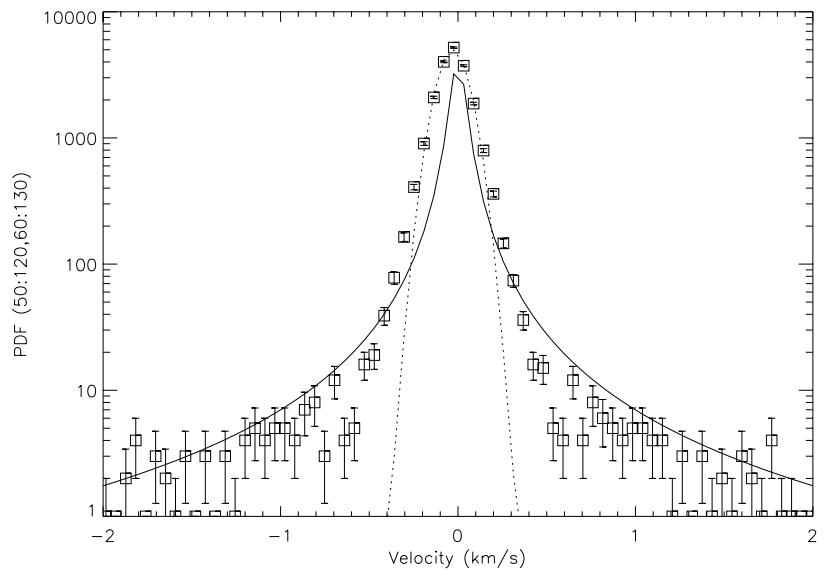

Fig. 11. Unnormalized fluctuation PDF from the ${ }^{12} \mathrm{CO}(1-0)$ data for the northern portion removing a smoothed ( 20 points) map as described in the text. This is compared with Gaussian (dashed line) and lorentzian (dotted line) functions.

lower left position $(50,50)$ to the upper right $(150,150)$ corner, corresponding to an angular area of $82 \operatorname{arcmin} \times 82$ arcmin for which the $\mathrm{S} / \mathrm{N}$ ratio is uniformly the highest in our map. The regions used for the velocity comparison were selected based on the integrated intensity of the line, retaining only those positions for which the integrated intensity was set to various levels $\geq 10 \mathrm{~K} \mathrm{~km} \mathrm{~s}^{-1}$, the minimum corresponding to a $\mathrm{S} / \mathrm{N}$ ratio of 10 for the relevant channels using the three channel smoothing for the individual line profiles.

For this subregion we first applied the method advocated by MSB99 and subtracted from the centroid map a smoothed 


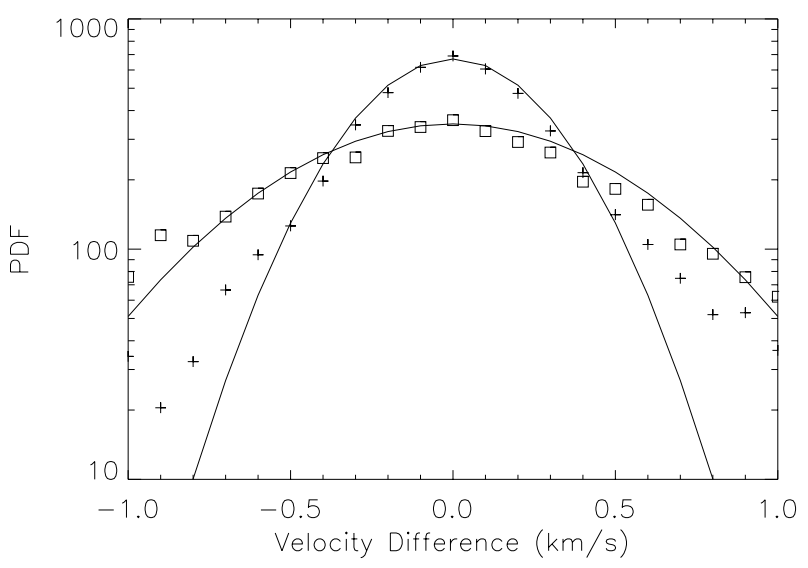

Fig. 12. Un-normalized shift PDF for the northern portion of the cloud $\left(100 \times 100 \mathrm{pix}^{2}\right.$, taken along $\Delta l=1$ (squares) and 5 (crosses) pixels, showing the relevant Gaussian distribution for each. Note the rapid convergence to a Gaussian profile that, as discussed in the text, indicates that the correlation scale is between 0.03 and $0.15 \mathrm{pc}$ for MBM 3. Note also the increasing width of the PDF with increasing lag, the result of the large scale flow visible in this region (see Fig. 10 and discussion in text).

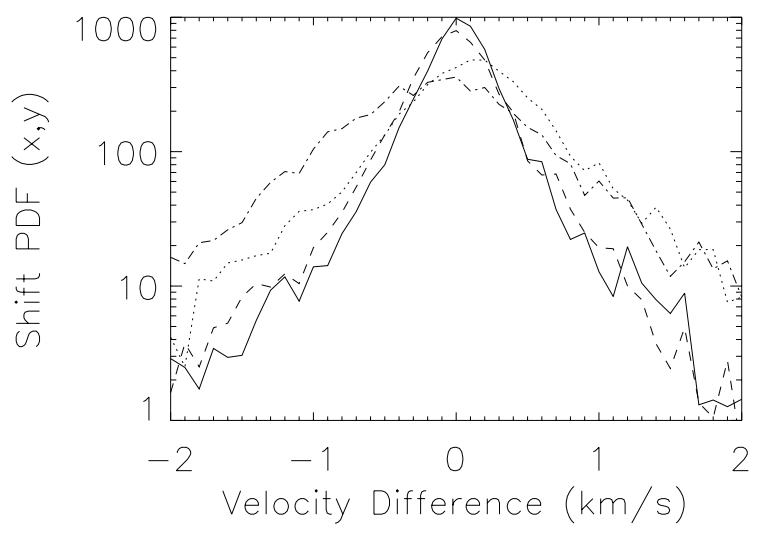

Fig. 13. Un-normalized shift PDF for the northern portion of the cloud. Lags were taken separately for $\Delta b$ and $\Delta l$, displaying the anisotropy introduced by the shear flow in this region of the map. The PDFs are shown for lags in $l$ of one pixel (solid) and 5 pixels (dotted)), and in $b$ for one (dashed), and 5 pixels (dot-dashed). Particularly notable is the difference in the extension of the PDF for the larger $b$ displacement.

map to remove the large scale structure, leaving a map of the centroid fluctuations, by using boxcar smoothings over a range between 15 and 30 pixels; for display we show the result for 20 point smoothing but the results were unchanged for smoothing above 15 points. The PDF, plotted in Fig. 11, was then constructed from the histogram of the residuals binned with the velocity resolution, $0.056 \mathrm{~km} \mathrm{~s}^{-1}$, with the error bars from Poisson statistics. The function still displays some asymmetry caused by an incomplete removal of the large scale gradient, a feature also seen in published studies of other clouds. For comparison we show the best fit Gaussian for which $\sigma=0.09 \mathrm{~km} \mathrm{~s}^{-1}$.

We also constructed velocity shift PDFs, that retain all the large-scale structure, by subtracting from the original centroid velocity map a copy that was shifted in two different ways. The first, shown in Fig. 12, used identical shifts of one beam in both Galactic latitude and longitude (thus the shift is actually 1.4 times the shift in each coordinate); this scalar lag is the one conventionally used in studies of molecular clouds where there are no large scale systematic flows. The figure shows the result

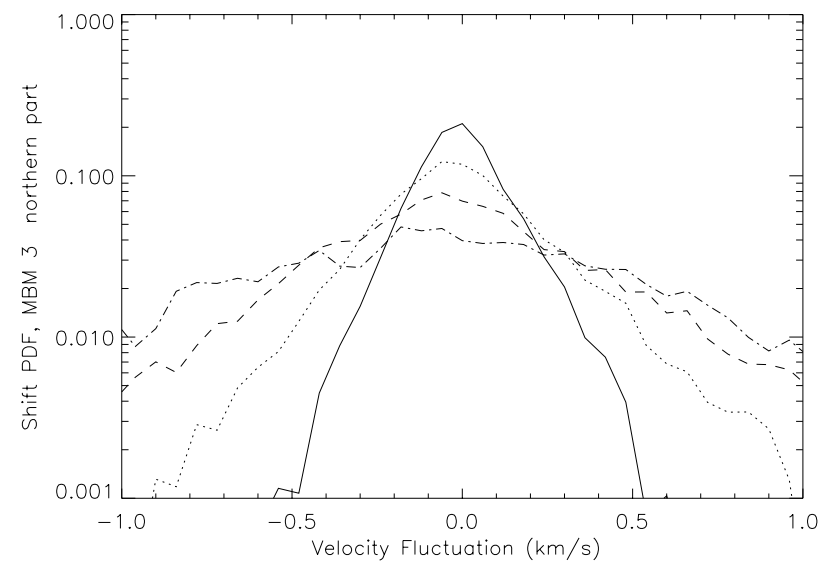

Fig. 14. Comparison of normalized shift PDFs for the northern portion of the cloud taken simultaneously in $(l, b)$ with 1 (solid), 2 (dotted), 4 (dashed), and 8 lags (dot-dashed) in each coordinate. The PDF is normalized by its integral.

for the fit of a Gaussian ( $\sigma=0.08 \mathrm{~km} \mathrm{~s}^{-1}$, similar to the differenced PDF) and, for comparison, we show a lorentzian profile, $\left(\delta v^{2}+a^{2}\right)^{-1}$ with $a=0.05 \mathrm{~km} \mathrm{~s}^{-1}$. We remark that for this PDF we have used only those points for which the integrated intensity was $\geq 100 \mathrm{~K} \mathrm{~km} \mathrm{~s}^{-1}$ to select only the very highest $\mathrm{S} / \mathrm{N}$ ratio data following the discussion by Pety \& Falgarone (2003). Note that the lorentzian fits quite well, especially in the wings of the PDF although the data lies between the two extremes, a point to which we will return shortly (see Sect. 4). The second analysis, performed by shifting separately in Galactic latitude and longitude as a test of isotropy, is shown in Fig. 13. These shifts were an attempt to avoid what we found is a significant residual from the large scale flow when the scalar lags are used over large distances, a feature that seems to appear as well in some of the published studies of clouds. Instead, this differentially shifted PDF approaches a Gaussian for lags exceeding 4 or 5 pixels, while for lags between 1 and 2 pixels, the non-Gaussian wings persist.

We also ran a series of tests to determine how centroid velocities depend on the $\mathrm{S} / \mathrm{N}$ threshold used for the weighting. We find no difference in centroids for thresholds between 10 and $40 \mathrm{~K} \mathrm{~km} \mathrm{~s}^{-1}$ for the integrated maps. In addition, we checked the robustness of the shift PDF by including normally distributed errors throughout the velocity map consistent with Gaussian fits to the individual line profiles in the region near the peak of the intensity map. Using a range of dispersions, from 1 to $10 \sigma$ (the mean error in the individual Gaussian fits was $0.01 \mathrm{~km} \mathrm{~s}^{-1}$ ) we find no change in our conclusions about the reality of the nonGaussian wings until the simulated dispersions are unacceptably large, $>5 \sigma$, about $0.1 \mathrm{~km} \mathrm{~s}^{-1}$ above which the PDF is dominated by the Gaussian distribution.

Figure 14 shows the convergence of the shift PDFs to a Gaussian for the longitudinal displacements. This behavior was also found by Pety \& Falgarone (2003) in non-star-forming clouds in the vicinity of star-less cores. The velocity difference PDF's constructed for a sample of 12 star-forming molecular clouds by MSB99 also reveal non-Gaussian behavior in the wings. This suggests that such behavior may be common in both the diffuse and dense ISM. An alternative approach, using a scalar shift for the large lags without removing the large scale gradient resulted in persistent wings whose width merely reflected the large scale structure, hence our justification for performing the shifts separately. 


\subsection{Linewidth-size relation}

A scaling relation between size and velocity dispersion within a region $L, \delta v \sim L^{\alpha}$ was first introduced by Larson (1981), who found $\alpha \approx 0.4$. Being close to $1 / 3$, this early result was expected for the structure function of a cascade (e.g. McComb 1992) and seemed to fit expectations of turbulence theory. Subsequent studies based on large-scale surveys (e.g., Dame et al. 1986 and Solomon et al. 1987) which included substantially larger numbers of clouds found $\alpha=0.5 \pm 0.05$. After these publications the existence of the size-velocity dispersion relation is much less clear.

The initial studies were categorized according to Goodman et al. (1998) as type II studies, those that combine several clouds using a single molecular tracer. The definition of cloud size was based on a minimum contour level, in combination with reasonably low noise, and spanned sizes from $\approx 1$ to $\approx 100 \mathrm{pc}$. More recent type II studies find a much weaker correlation with flatter slopes $(\alpha=0.31 \pm 0.07$, Kawamura et al. 1998; $0.26 \pm 0.09$, Yonekura et al. 1997). However, as noted by Yonekura et al. (1998) cloud radii in these studies was defined by $50 \%$ of peak contour and only clouds and clumps with sizes less than $6 \mathrm{pc}$ were considered. These results are consistent with the study of Simon et al. (2001) who used an objective algorithm GAUSSCLUMPS (Stutzki \& Gusten 1990) to identify clumps in the BU-FCRAO Galactic Ring Survey. Although such algorithms can introduce biases (see Schneider \& Brooks 2005) Simon et al. (2001), who concentrated on clouds smaller than about $5 \mathrm{pc}$, find a much flatter index or no relation at all.

On scales of order 0.1 to 1 pc the results have been inconsistent. Loren (1989) found no relation for a large sample of clumps in $\rho$ Oph that spanned this range of sizes. Caselli \& Myers (1995) analyzed high density cores and found $\alpha=0.23 \pm 0.03$ for high mass cores and $0.53 \pm 0.07$ for low mass cores. Peng et al. (1998) find that the relationship holds for 0.02 to $0.04 \mathrm{pc}$ cores in the Taurus clouds but Goodman et al. (1998), who examined $\mathrm{NH}_{3}$ cores, showed that at the scale of about 0.1 pc these cores make a transition from turbulent to thermal support, indicating a breakdown in the relation at this scale. More recently, Onishi et al. (2002) find no relation for a large sample of cores in Taurus with sizes of order $0.1 \mathrm{pc}$, and Tachihara et al. (2002), in the largest sample of cores to date (136 star-less cores spanning a range of size from 0.06 to $0.4 \mathrm{pc}$ from several different molecular cloud complexes), find no statistically significant linewidth-size scaling. Thus the majority of the observational studies find that the linewidth-size relation does not hold for scales less than $1 \mathrm{pc}$.

The only unambiguous scaling relation emerges when clouds of large size are included. This point is substantiated by the recent study of Heyer \& Brunt (2004) who apply the technique of principal component analysis to molecular clouds in the widefield imaging surveys of the FCRAO. They find a very tight correlation with $\alpha=0.62 \pm 0.09$ for clouds between 3 and $30 \mathrm{pc}$. Although the PCA is a different approach from the techniques used in the previously cited studies, the result is consistent with the finding that the relationship is better defined for larger clouds.

In contrast to high density cores, translucent clouds are low density objects on scales sizes of a few pc. Based on a sample of 29 clouds, although limited in spatial scale, (sizes were determined by $2 \sigma$ contour) Heithausen (1996) finds that translucent clouds appear to lie on the extension of the relationship defined for larger clouds. In an earlier study of the translucent cloud MBM16 (LaRosa et al. 1999) we found several regions within the cloud whose centroid dispersions were anomalously
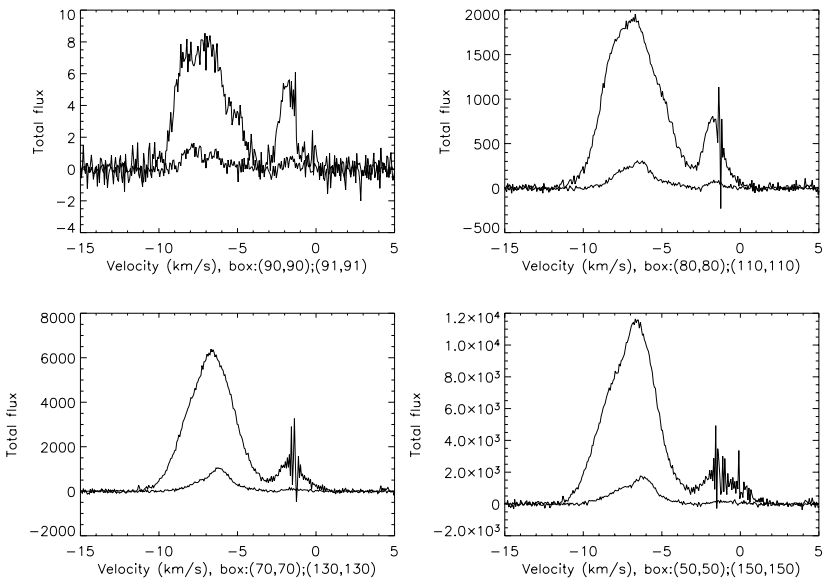

Fig. 15. Summed profiles for increasingly extensive regions of MBM 3 in ${ }^{12} \mathrm{CO}(1-0)$ (stronger) and ${ }^{13} \mathrm{CO}(1-0)$ (weaker) in the velocity range -10 to $8 \mathrm{~km} \mathrm{~s}^{-1}$.

small and consequently found no velocity dispersion size relation for substructures in this cloud. To eliminate bias when assigning sizes we follow earlier procedures (LaRosa et al. 1999; Ossenkopf \& Mac Low 2002) and sum profiles over progressively larger regions surrounding an arbitrarily chosen position. We find no correlation between velocity width and size of the sampled region in MBM3. In Fig. 15 we show how the summed profiles change with the size of the sampled region. The FWHM of a single profile is only marginally less than the profiles summed over the entire cloud. This result spans a factor of 50 in size and calls into question the universality of the linewidth-size relation. The relationship between this diagnostic and velocity PDFs is discussed in detail in the next section.

\section{Discussion}

\subsection{The Larson law and large scale flows}

The linewidth-size relation and its breakdown at small scales suggests the following interpretation. There are along any line of sight in the ISM random and systematic motions. Linewidths alone cannot be used to distinguish these. Since, however, systematic motions and gradients are detected on a variety of size scales from velocity centroid maps, when these gradients are large enough relative to the intrinsic velocity dispersion they will dominate the linewidths. As the surveyed area increases, the probability of encountering large gradients increases; thus, the linewidth must increase. This may explain why the linewidthsize relation is better defined for clouds with sizes larger than $10 \mathrm{pc}$. In smaller clouds, the linewidth is dominated by smaller scale random motions, since it is unlikely such clouds will have more than a single velocity gradient, and the velocity dispersion will no longer depend on the size of the sample or its location in the cloud. There is, however, an intrinsic connection between these two flows: velocity shears and gradients are often unstable, transforming ordered into random motions and if a cascade develops, any large scale gradients are smeared out, leaving only small scale centroid fluctuations. Although a randomizing process, the mark of turbulence is not just a dispersion or fluctuation in any or all velocity components. The statistical quantity, variance, measures only the range over which motions may be present. Only with all of the moments of a distribution is it possible to distinguish random flows from turbulence. Although in MBM 3 we find the same variance independent of the position 
in the cloud and independent of the area of the measured region, the PDF of the centroids is governed by non-Gaussian processes. The velocity fluctuations are correlated on some unresolved scale, which is the robust evidence for turbulence in any laboratory or terrestrial flow (Minier \& Peirano 2001). In this interpretation the linewidth-size relation is not disclosing a cascade although it is certainly sensitive to flows on many scales, some of which may ultimately drive the turbulence.

\subsection{Intermittency and PDFS}

The definitive property of turbulent motions is intermittency. This can be defined as the occurrence of large amplitude, rare events with much greater frequency than a Gaussian (uncorrelated) process. A power law is one example of stable, stationary probability distribution function even though the first and second moments are undefined. Established as a class of functions satisfying statistical independence and the probability axioms, called Lèvy distributions, such PDFs cannot in general be expressed in closed form (e.g. Feller 1971; Sornette 2004). However, one such function, the Cauchy distribution, is well known as a closed form (a lorentzian function) and is a special case of a Lèvy distribution for an exponent of 1 . Our choice of this form for matching the PDF, shown in Fig. 6, is motivated by this observation. It is becoming increasingly clear that Lèvy distributions are far from rare in cosmic environments. The various non-Gaussian forms for luminosity and mass functions of galaxies, stars, and dust - e.g. Salpeter or Kroupa mass functions, Schechter luminosity function, etc. - are almost all marked by some range in which they are scale free - that is, power laws. Recently it was proposed that a Lèvy distribution produces the electron density fluctuations needed to explain the anomalous $\lambda^{4} D M^{4}$ scaling for temporal pulse broadening of pulsars as a function of wavelength $\lambda$, with dispersion measures, $D M$, exceeding $20 \mathrm{pc} \mathrm{cm}^{3}$. Boldyrev \& Gwinn (2003a,b, 2005) have derived the observed scaling assuming a power law electron density PDF.

As we noted in Sect. 3.4, with increasing lag, the central limit theorem guarantees that the fluctuations must become increasingly decorrelated so the PDF must approach a Gaussian. This convergence is also a measure of the correlation length independent of an explicit measurement of the correlation function. We note that while a Gaussian process can result from adiabatic (merely chaotic) processes, a Lèvy process cannot, and thus the smallest scales sampled by the shift PDFs (Lagrangian) for only a few lags, or from those obtained using smoothed centroid map differences (Eulerian) must be detecting the comparatively small, dense regions in which the cascade terminates and the kinetic energy dissipates.

\subsection{Energetics}

Classical turbulence theory requires that dissipation occurs as the energy cascades to a scale where molecular viscosity becomes important. In the ISM this scale is set by the neutralneutral and neutral-ion collision mean free path, $\approx 2 \times 10^{14} / \mathrm{n} \mathrm{cm}$ (Huba 2004), and is of order 0.01 AU for a number density $n=10^{3} \mathrm{~cm}^{-3}$ (a mean value derived using $N_{\mathrm{H}_{2}}$ assuming a thickness of order $0.5 \mathrm{pc}$ for the cloud that we will use subsequently for scaling). This is many orders of magnitude smaller than either the injection scale or any observable scale.

To compute the energetics of the turbulence we need some way to estimate appropriate scales of velocity and length. We have three characteristic sizes from the observations: a) the size of the whole cloud on which the systematic motions are observed; b) the correlation length obtained from the relaxation of the velocity centroid shift PDFs; and c) the distance over which the intensity of the line profiles changes significantly. The first is about $1 \mathrm{pc}$, the second is $\leq 0.1 \mathrm{pc}$ (see Sect. 4.2, and Fig. 12), and the last is about $0.1 \mathrm{pc}$. We detect significant changes in the line intensities over a scale of $\approx 0.1 \mathrm{pc}$, as SMLM03 reported for MBM 40. Thus if the typical size for structures is of the same order as their width projected on the sky the depth along the line of sight is also $0.1 \mathrm{pc}$.

Assuming a standard Kolmogorov cascade for a velocity dispersion of $\sigma_{v}$ at the associated length $l$, the energy transfer rate is $\epsilon_{\text {trans }} \sim \rho\left(\sigma_{v}\right)^{3} \ell^{-1}$ and we use $\ell \approx 0.1$ pc for the cascade. We can estimate $\sigma_{v}$ from the line profiles. Any single line profile samples the entire line of sight within a beam and for MBM 3 the FWHM gives $\sigma_{v} \approx 1.7 \mathrm{~km} \mathrm{~s}^{-1}$, independent of the location. Scaling to a density of $10^{3} \mathrm{~cm}^{-3}$, we have $\epsilon_{\text {trans }} \approx$ $3 \times 10^{-23} \mathrm{erg} \mathrm{s}^{-1} \mathrm{~cm}^{-3}$. If we assume instead that $\sigma_{v}$ is the FWHM of the one lag shift PDF, then for a single beam $\ell \approx 0.03 \mathrm{pc}$ and $\sigma_{v} \approx 0.4 \mathrm{~km} \mathrm{~s}^{-1}$ giving $\epsilon_{\text {trans }} \approx 3 \times 10^{-24} \mathrm{erg} \mathrm{s}^{-1} \mathrm{~cm}^{-3}$. In contrast, to estimate the rate of energy injection from the shear flow we suggest an approximation for the turbulent viscosity, $\eta_{\mathrm{T}}=\rho \ell \sigma_{v}$ by taking $\sigma_{v}$ from the line profiles and estimating $\ell$ with the correlation length. Then since $\epsilon_{\text {inj }}=\eta_{\mathrm{T}}(\Delta V / L)^{2}$ and our velocity gradient is $\Delta V / L \approx 3 \mathrm{~km} \mathrm{~s}^{-1} \mathrm{pc}^{-1}$, we find $\epsilon_{\text {inj }} \approx 10^{-24} \mathrm{erg} \mathrm{s}^{-1} \mathrm{~cm}^{-3}$. Based on numerical simulations of driven MHD turbulence, Juvela et al. (2001) used non-LTE radiative transfer to compute the $\mathrm{CO}$ cooling rate as a function of temperature and column density. Their results differ somewhat from earlier results obtained by Goldsmith \& Langer (1978) and Neufeld et al. (1995) since the optical depth is reduced by the turbulent motions. Density inhomogeneites are also computed selfconsistenly. For a temperature of $10 \mathrm{~K}$ and our observed column density of $7 \times 10^{20} \mathrm{~cm}^{-2}$ the CO cooling rate is $10^{-24} \mathrm{erg} \mathrm{s}^{-1} \mathrm{~cm}^{-3}$ so given the estimates for energy injection and transfer rates, the $\mathrm{CO}$ cooling may keep up with the turbulent energy production in this cloud.

\section{Conclusions}

We have presented a detailed analysis of the velocity field in the high latitude cirrus cloud MBM 3. The linewidths in this cloud are unusually large, one of the reasons we included it in our study. We find that the linewidth-size relation does not hold in this cloud and thus is not tracing only turbulent motions. However, non-Gaussian velocity PDFs are the robust diagnostic of intermittency and this is what we detect. We conclude, from the PDFs, that MBM 3 clearly displays turbulence and requires continued powering of the superthermal motions which can only be driven externally. As we found for MBM 16 and MBM 40, the source is large (parsec or more) scale shear flows in the atomic gas. Lastly we estimate the turbulent energy transfer rate produced as a consequence of these flows. For single line profiles the gas motions are always superthermal and for a density $10^{3} \mathrm{~cm}^{-3}$ yields a Jeans length of about $4 \mathrm{pc}$ which is much larger than the cloud; a density a factor of 100 higher would be required to induce instability on this scale. This suggests that MBM3 and similar low mass clouds will not become gravitationally unstable.

Acknowledgements. S.N.S. has been supported, in part, by funds from MIUR and INFN through the Dept. of Physics, Univ. of Pisa. T.N.L. has been supported by a grant from the Center for Excellence in Teaching and Learning at Kennesaw State Univ. T.N.L. also thanks the INFN/Pisa and the section director, Rino Castaldi, for travel support and the Astrophysics group at the University of 
Pisa for their warm hospitality. We thank Paola Caselli, Sergio Chibbaro, Paolo Grigolini, Mark Heyer, Alex Lazarian, Jean-Pierre Minier, Francesco Pegoraro, Nicholas Schneider, Enrique Vazquez-Semadeni and Ethan Vishniac for discussions and papers, the anonymous referee for a very helpful and civil review, and Francesco Califano for advice on producing the accompanying movie in IDL. This study has used data from the IPAC IRAS archives and the bibliographic data from the ADS.

\section{Appendix}

Online Material: Movie of ${ }^{12} \mathrm{CO}$ (1-0) Channel Maps for MBM 3. The movie shows the structure of the cloud in the ${ }^{12} \mathrm{CO}(1-0)$ line in the radial velocity interval $-11.36 \leq v_{\mathrm{rad}} \leq$ $-3.68 \mathrm{~km} \mathrm{~s}^{-1}$ in steps of $0.064 \mathrm{~km} \mathrm{~s}^{-1}$ per frame (channels 340 to 450) for the intensity interval $0.1 \leq T_{\mathrm{A}} \leq 3 \mathrm{~K}$. Galactic north is up, Galactic longitude increases to the left. The second component, shown in the integrated line profiles (visible e.g. in Fig. 10) is not shown. On the eastern side of the cloud, the filamentary sheared structures we describe in the text are clearly visible, near the center of the cloud and toward the north. Notice that the cloud emission appears at almost the same velocity but persists over a broader velocity range in the northern part of the cloud. Flows extend toward both east and west in the later channels, extending toward the west in the same direction as the IRAS structures (see Fig. 1). A small component to the south also connects to one of an IRAS structure.

\section{References}

Bazell, D., \& Desert, F. X. 1990, ApJ, 333, 353

Bohlin, R. C., Savage, B. D., \& Drake, J. F. 1978, ApJ, 224, 132

Boldyrev, S., \& Gwinn, C. 2003a, Phys. Rev. Lett., 91, 131101

Boldyrev, S., \& Gwinn, C. 2003b, ApJ, 584, 791

Boldyrev, S., \& Gwinn, C. 2005, ApJ, 624, 213

Caselli, P., \& Myers, P. C. 1995, ApJ, 446, 665

Chastain, R. 2005, Ph.D. Thesis/Physics and Astronomy, Univ. of Georgia

Corbelli, E., Palla, F., \& Zinnecker, H. 2005, The Initial Mass Function 50 years Later (Dordrecht: Springer)

Dame, T. M., Elmegreen, B. G., Cohen, R. S., \& Thaddeus, P. 1986, ApJ, 305, 892

Dickman, R. L. 1978, ApJS, 37, 407

Elmegreen, B. G., \& Scalo, J. 2004, ARA\&A, 42, 211

Falgarone, E., \& Phillips, T. G. 1990, ApJ, 359, 344

Falgarone, E., \& Passot, T. 2003, Turbulence in Magnetic Fields in Astrophysics: Lecture Notes in Physics, 614 (Dordrecht: Springer)

Falgarone, E., Panis, J. F., Heithausen, A., et al. 1998, A\&A, 331, 669

Feller, W. 1971, An Introduction to Probability Theory and its Applications, Vol. II (New York: Wiley)

Gautier, T. N., Boulanger, F., Perault, M., \& Puget, J. L. 1992, AJ, 103, 1313

Goldsmith, P. F., \& Langer, W. D. 1978, ApJ, 222, 881

Goodman, A. A., Barranco, J. A., Wilner, D. P., \& Heyer, M. H. 1998, ApJ, 504, 223
Gomez de Castro, A. I., Heyer, M., Vazquez-Semadeni, E., et al. 2004, Magnetic Fields and Star Formation: Theory versus Observations (Dordrecht: Kluwer)

Hartmann, D., \& Burton, W. B. 1997, Atlas of Galactic Neutral Hydrogen (Cambridge, UK: Cambridge Univ. Press)

Heyer, M. H., \& Brunt, C. M. 2004, ApJ, 615, L45

Heithausen, A. 1996, A\&A, 314, 251

Huba, J. D. 2004, Naval Research Laboratory Plasma Formulary (Washington DC: Naval Research Laboratory)

Ingalls, J. G., Bania, T. M., Lane, A. P., Rumitz, M., \& Stark, A. A. 2000, ApJ, 535,211

Juvela, M., Padoan, P., \& Nordlund, Å. 2001, ApJ, 563, 853

Juvela, M., Padoan, P., \& Jimenez, R. 2003, ApJ, 591, 258

Kitamura, Y., Sunada, K., Hayashi, M., \& Hasegawa, T. 1993, ApJ, 413, 221

Kawamura, A., Onishi, T., Yonekura, Y., et al. 1998, ApJS, 117, 387

Lallement, R., Welsh, B. Y., Vergely, J. L., Crifo, F., \& Sfeir, D. 2003, A\&A, 411,447

Langer, W. D., \& Penzias, A. A. 1990, ApJ, 408, 539

LaRosa, T. N., Shore, S. N., \& Magnani, L. 1999, ApJ, 512, 761

Larson, R. B. 2003, Rep. Prog. Phys., 66, 1651

Lis, D. C., Pety, J., Phillips, T. G., \& Falgarone, E. 1996, ApJ, 463, 623

Loren, R. B. 1989, ApJ, 338, 925

Low, F. J., Young, E., Beintema, D. A., et al. 1984, ApJ, 278, L19

Mac Low, M.-M., \& Klessen, R. S. 2004, Rev. Mod. Phys., 76, 125

Magnani, L., Blitz, L., \& Mundy, L. 1985, ApJ, 295, 402

Magnani, L., Hartmann, D., Holcomb, S. L., Smith, L. E., \& Thaddeus, P. 2000, ApJ, 535, 167

Magnani, L., Chastain, R. J., Kim, H. K., et al. 2003, ApJ, 586, 1111

McComb, W. D. 1992, The Physics of Fluid Turbulence (London: Oxford Univ. Press)

Miesch, M. S., \& Bally, J. 1994, ApJ, 429, 625

Miesch, M. S., Scalo, J., \& Bally, J. 1999, ApJ, 524, 895

Minier, J., \& Peirano, E. 2001, Phys. Rep., 352, 1

Neufeld, D. A., Lepp, S., \& Melnick, G. J. 1995, ApJS, 100, 132

Onishi, T., Mizuno, A., Kawamura, A., Tachihara, K., \& Fukui, Y. 2002, ApJ, 575,950

Ossenkopf, V., \& Mac Low, M.-M. 2002, A\&A, 390, 307

Peng, R., Langer, W. D., Velusamy, T., Kuiper, T. B. H., \& Levin, S. 1998, ApJ, 497,842

Pety, J., \& Falgarone, E. 2003, A\&A, 412, 417

Pope, S. B. 1994, Ann. Rev. Fluid Mech., 26, 23

Scalo, J., \& Elmegreen, B. G. 2004, ARA\&A, 42, 275

Schlegel, D. J., Finkbeiner, D. P., \& Davis, M. 1999, ApJ, 500, 525

Schneider, N., \& Brooks, K. 2005, PASA, 21, 290

Shore, S. N., Magnani, L., LaRosa, T. N., \& McCarthy, M. M. 2003, ApJ, 593, 413 (SMLM03)

Simon, R., Jackson, J. M., Clemens, D. P., Bania, T. M., \& Heyer, M. H. 2001, ApJ, 551, 747

Solomon, P. M., Rivolo, A. R., Barret, J., \& Yahil, A. 1987, ApJ, 319, 730

Sornette, D. 2004, Critical Phenomena in the Natural Sciences (Berlin: SpringerVerlag)

Sreenivasan, K. R., \& Antonia, R. A. 1997, Ann. Rev. Fluid Mech., 29, 435

Stutzki, J., \& Guesten, G. 1990, ApJ, 356, 513

Tachihara, K., Onishi, T., Mizuno, A., \& Fukui, Y. 2002, A\&A, 385, 909

Tenenkes, H., \& Lumley, J. L. 1972, A First Course in Turbulence (Cambridge, MA: MIT Press)

Weiland, J. L., Blitz, L., Dwek, E., et al. 1986, ApJ, 306, L101

Wheelock, S. L., et al. 1994, IRAS Sky Survey Explanatory Supplement, JPL Publication 94-11 (Pasadena: JPL)

Yonekura, Y., Dobashi, K., Mizuno, A., Ogawa, H., \& Fukui, Y. 1997, ApJS, 110,21 